\title{
The ubiquitous nature of the horizontal branch second $U$-jump
}

\section{A link with the Blue Hook scenario?^}

\author{
Y. Momany ${ }^{1}$, L. R. Bedin ${ }^{1}$, S. Cassisi ${ }^{2}$, G. Piotto ${ }^{1}$, S. Ortolani ${ }^{1}$, A. Recio-Blanco ${ }^{1}$, F. De Angeli ${ }^{1}$, and F. Castelli ${ }^{3}$ \\ 1 Dipartimento di Astronomia, Università di Padova, Vicolo dell’Osservatorio 2, 35122 Padova, Italy \\ e-mail: [piotto, bedin, ortolani, recio, deangeli]@pd.astro.it \\ 2 INAF - Osservatorio Astronomico di Collurania, Via M. Maggini, 64100 Teramo, Italy \\ e-mail: cassisi@te.astro.it \\ 3 INAF - Osservatorio Astronomico di Trieste, via Tiepolo 11, 34131 Trieste, Italy \\ e-mail: castelli@ts.astro.it
}

Received 17 November 2003 / Accepted 19 February 2004

\begin{abstract}
In a previous paper we reported on a discontinuity in the extreme horizontal branch (EHB) of the Galactic globular cluster NGC 6752, which we called the second $U$-jump. This feature was attributed to a combination of post zero-age horizontal branch evolution and diffusion effects. In this follow-up study we analyze other EHB clusters and show that the second $U$-jump is a common feature among EHB clusters reaching $T_{\text {eff }} \geq 23000 \mathrm{~K}$, and that its onset in different clusters converges around $T_{\text {eff }} \sim 21000 \pm 3000 \mathrm{~K}$. We also present near-ultraviolet diagrams of $\omega$ Cen and NGC 2808 , the only two objects with spectroscopically confirmed "blue hook" stars $\left(T_{\text {eff }} \geq 35000 \mathrm{~K}\right)$. We confirm predictions of a photometric discontinuity separating late from early-helium flashers. Moreover, we present empirical evidence that the second $U$-jump population might be mainly composed of early-helium flashers. Lastly, we revisit the discussion on the ubiquitous nature of the gaps and jumps so far identified in the blue HB tails, suggesting a possible discrete nature of the distribution in temperature of the HB stars.
\end{abstract}

Key words. stars: horizontal-branch - stars: Hertzsprung-Russell (HR) and C-M diagrams - stars: Population II Galaxy: globular clusters: general - ultraviolet: stars

\section{Introduction}

Over the last decades, both observational and theoretical efforts have been devoted to the analysis of the observed distribution of stars along the Horizontal Branch (HB) of Galactic globular clusters. This notwithstanding, our understanding of several observational features is still incomplete. Even if theory and observations agree that the HB morphology is governed by metallicity (the first parameter), since the late sixties it has become clearer that the color distribution of HB stars in Galactic globular clusters is not a unique function of metallicity (Sandage \& Wildey 1967). NGC 362 and NGC 288 are a classical example of how two clusters sharing similar metallicities can show remarkably different HB morphology. Hence, other parameters besides metallicity (e.g. age, cluster environment, helium abundance, mass loss and rotation) affect the evolution of HB stars (second parameter debate).

The second parameter effect is not the only puzzling feature in the evolution of HB stars. In particular, photometric studies

Send offprint requests to: Y. Momany, e-mail: momany@pd . astro.it

* Based on observations with the ESO/MPI $2.2 \mathrm{~m}$ and ESO/NTT telescopes, located at La Silla Observatory (Chile) and on observations with the NASA/ESA Hubble Space Telescope. of stars hotter than the RR Lyrae instability strip showed the presence of: (a) gaps along the blue tail (Ferraro et al. 1998; Piotto et al. 1999); (b) a jump around $T_{\text {eff }} \sim 11500 \mathrm{~K}$ in the Strömgren $u, u-y$ (Grundahl et al. 1999, hereafter G99) and Johnson $U, U-V$ (Bedin et al. 2000) color-magnitude diagrams (CMDs); (c) hot HB stars reaching temperatures of $T_{\text {eff }} \simeq 30000 \mathrm{~K}$ or more in metal-poor (D'Cruz et al. 1996; Brown et al. 2001) and metal-rich (Rich et al. 1997) clusters; and (d) the still unexplained presence of fast $\mathrm{HB}$ rotators (Behr et al. 2000; Recio-Blanco et al. 2002). On the other hand, spectroscopic studies showed abundance (Behr et al. 1999) and gravity anomalies (Moehler et al. 2000) in stars hotter than $T_{\text {eff }} \sim 11500 \mathrm{~K}$.

Horizontal branch stars hotter than $T_{\text {eff }} \sim 20000 \mathrm{~K}$ are usually referred to as extreme $\mathrm{HB}$ (EHB) stars. It is believed that EHBs experience high mass-loss during their red giant phase, reducing their $\mathrm{H}$-rich envelope down to $\leq 0.05 M_{\odot}$, to the point of being unable to sustain H-shell burning. However, it is hard to explain why such an enhanced mass loss occurs along the red giant branch. Near-UV CMDs of NGC 6752 (Momany et al. 2002) have revealed another interesting feature along the EHB. In the $U$ vs. $(U-V)$ plane, the HB showed a discontinuity at $U-V \simeq-1.0$ (corresponding $T_{\text {eff }} \sim 23000 \mathrm{~K}$ ). 
Table 1. Ground-based observation log along with the HST archival data.

\begin{tabular}{|c|c|c|c|c|c|}
\hline Object & Instrument & Prog. ID & Date & Filters & Seeing \\
\hline NGC 1904 & WFI@2.2 & 64.L-0255 & 1999 & $U, B, V$ & $0.8-1{ }^{\prime \prime} 2$ \\
\hline NGC 6752 & WFI@2.2 & 65.L-0561 & 2000 & $U, B, V$ & $0 .{ }^{\prime} 6-0.9$ \\
\hline NGC 7099 & WFI@2.2 & 65.L-0561 & 2000 & $U, B, V$ & $0.6-0.9$ \\
\hline NGC 6273 & WFI@2.2 & 65.L-0561 & 2000 & $U, B, V$ & $0.7-1 . \prime 1$ \\
\hline NGC 7089 & WFI@2.2 & 69.D-0582 & 2002 & $U, B, V, I$ & $0.7-1 . .3$ \\
\hline NGC 5139 & WFI@2.2 & 69.D-0582 & 2002 & $U, B, V, I$ & $0.6-1 . .5$ \\
\hline NGC 5986 & SUSI2@NTT & 71.D-0175 & 2003 & $U, V$ & $0.9-1 . .4$ \\
\hline NGC 6656 & SUSI2@NTT & 71.D-0175 & 2003 & $U, V$ & $0.9-1 . \prime 4$ \\
\hline NGC 6715 & SUSI2@NTT & 71.D-0175 & 2003 & $U, V$ & $0.9-1 . .4$ \\
\hline NGC 2808 & WFPC2@HST & GO8655 & 2001 & $F 450 W$ & \\
\hline NGC 2808 & WFPC2@HST & GO6804 & 1998 & $F 336 W$ & \\
\hline NGC 6093 & WFPC2@HST & GO8655 & 2000 & F450W & \\
\hline NGC 6093 & WFPC2@HST & GO6460 & 1997 & $F 336 W$ & \\
\hline NGC 6205 & WFPC2@HST & GO5903 & 1996 & $F 336 W, F 450 W, F 555 W$ & \\
\hline
\end{tabular}

Given the apparent photometric similarities with the "cooler" G99 jump, and the clear difference in temperature with respect to blue hook stars (i.e. blue hook stars are generally hotter than $T_{\text {eff }} \sim 35000 \mathrm{~K}$ ), we called this feature the "second$U$ jump", and tentatively attributed it to a combination of post ZAHB evolution and diffusion effects.

Most of these puzzling features remain unsolved. In particular, we lack a global view on the origin and internal properties of EHB stars. This is not a problem confined to the final stages of evolution of globular cluster stars. Indeed, the nature of EHBs has a more general relevance in astrophysics as these are considered responsible for the UV excess observed in the spectra of elliptical galaxies ( $U V$-upturn galaxies, Greggio \& Renzini 1990).

In this paper we present new near-UV CMDs for a selected sample of Galactic globular clusters, characterized by an HB with an extended blue tail, to investigate the observational properties of the EHB stars in these clusters. In the following section we discuss the observational data-base and briefly outline the main reduction and calibration procedures; in Sect. 3 we show that the second $U$-jump, already identified in NGC 6752, is also present in other EHB clusters; in Sect. 5 we suggest a link between the second $U$-jump feature and the He flash-induced mixing scenario discussed by Brown et al. (2001). A summary will close the paper.

\section{Observations and data reduction}

In Table 1 we report the observation log of all data used in this paper, while in Table 2 we list the main properties, such as metallicity, reddening, apparent distance moduli and concentration, for the selected clusters. Here follows a short description of the data reduction process.

\subsection{Ground-based data}

The ground-based data consist of $U B V I$ observations obtained in three different runs with the Wide-Field Imager (WFI) at the $2.2 \mathrm{~m}$ ESO-MPI telescope and one run using SUSI2 at the ESO-NTT (both telescopes are located in La Silla, Chile).

The WFI camera consists of eight $2048 \times 4096$ EEV-CCDs, with a total field of view of $34 \times 33 \mathrm{arcmin}^{2}$. The exposure times were divided in deep and shallow to sample both bright red giants and the faint main sequence and HB stars (e.g. the $U$ images of NGC 2808 were taken as a series of 180 and $2000 \mathrm{~s}$ exposures). All scientific images were dithered so as to cover the gaps separating the eight $2048 \times 4096 \mathrm{CCDs}$. The seeing conditions were generally good during the three runs (a typical run consisted of 3 nights). Shallow exposures were obtained in photometric nights, and these were used to calibrate the deep data obtained in conditions of thin cirrus. Basic reductions of the CCD mosaic (de-biasing and flat-fielding) were performed using the IRAF package MSCRED (Valdes 1998).

Stellar photometry was performed using the DAOPHOT and ALLFRAME programs (Stetson 1994). For a detailed presentation we refer the reader to Momany et al. (2002) and Bedin et al. (2000). To perform stellar photometry for 8 CCDs, different DAOPHOT/ALLFRAME tasks were included in routines to automatize the reduction process. This pseudo photometric-pipeline performed (1) the construction of the point spread function (PSF) for each single image; (2) initial fitting photometry; (3) an estimate of the aperture-correction from curves of growth of the brightest stars; (4) the construction of median image for each chip, and the creation of the master list of star-like objects; and (5) PSF fitting of the master list on each single exposure. The deep and shallow catalogs were then matched, and a final catalog in each passband was obtained. To reduce the identification of spurious objects, we imposed that objects recorded in the final catalog of each passband must have been identified in at least half of the single exposures (i.e. when merging $6 V$ catalogs, only objects identified in at least 3 images were registered). The instrumental magnitudes were normalized to $1 \mathrm{~s}$ exposure and zero airmass. Finally, the PSF magnitudes were converted into aperture magnitudes assuming that $m_{\mathrm{ap}}=m_{\mathrm{PSF}}-$ constant, where the constant is the the aperture correction. 
Table 2. Properties of cluster sample. Columns 2 to 5 are from the Harris on-line-catalog: http://physun.physics.mcmaster.ca/ harris/mwgc. dat (1996) as updated on February 2003. Columns 7 and 8 are from Rosenberg et al. (1999).

\begin{tabular}{|c|c|c|c|c|c|c|c|c|c|}
\hline Object & $E_{B-V}$ & $(m-M)_{V}$ & {$[\mathrm{Fe} / \mathrm{H}]$} & $c^{1}$ & $M_{V}$ & $\Delta V_{\mathrm{TO}}^{\mathrm{HB}}$ & Normalized relative age & Second $U$-jump & Blue hook \\
\hline NGC 6752 & 0.04 & 13.13 & -1.56 & $\mathrm{c}$ & -7.73 & 3.55 & 1.03 & $\mathrm{y}$ & $\mathrm{n}$ \\
\hline NGC 6656 & 0.34 & 13.60 & -1.64 & 1.31 & -8.50 & 3.55 & 1.04 & $\mathrm{y}$ & $\mathrm{n}$ \\
\hline NGC 5139 & 0.12 & 13.97 & $-1.62^{2}$ & 1.61 & -10.29 & - & - & $\mathrm{y}$ & $\mathrm{y}$ \\
\hline NGC 6205 & 0.02 & 14.48 & -1.54 & 1.51 & -8.70 & 3.55 & 1.02 & $\mathrm{y}$ & $y^{3}$ \\
\hline NGC 7099 & 0.03 & 14.62 & -2.12 & $\mathrm{c}$ & -7.43 & - & - & $\mathrm{n}$ & $\mathrm{n}$ \\
\hline NGC 288 & 0.03 & 14.83 & -1.24 & 0.96 & -6.74 & 3.55 & 0.97 & $\mathrm{n}$ & $\mathrm{n}$ \\
\hline NGC 7089 & 0.06 & 15.49 & -1.62 & 1.80 & -9.02 & - & - & $\mathrm{y}$ & $\mathrm{n}$ \\
\hline NGC 6093 & 0.18 & 15.56 & -1.75 & 1.95 & -8.23 & 3.55 & 1.04 & $\mathrm{y}$ & $\mathrm{n}$ \\
\hline NGC 2808 & 0.22 & 15.59 & -1.15 & 1.77 & -9.39 & 3.30 & 0.81 & $\mathrm{n}$ & $\mathrm{y}$ \\
\hline NGC 1904 & 0.01 & 15.59 & -1.57 & 1.72 & -7.86 & 3.50 & 1.00 & $\mathrm{n}$ & $\mathrm{n}$ \\
\hline NGC 6273 & $0.41^{4}$ & 15.95 & -1.68 & 1.53 & -9.18 & - & - & $\mathrm{y}$ & $\mathrm{n}$ \\
\hline NGC 5986 & 0.28 & 15.96 & -1.58 & 1.22 & -8.44 & - & - & $\mathrm{y}$ & $\mathrm{n}$ \\
\hline NGC 6715 & 0.15 & 17.61 & $-1.58^{2}$ & 1.84 & -10.01 & - & - & $\mathrm{y}$ & $y^{3}$ \\
\hline
\end{tabular}

${ }^{1}$ Central concentration, $c=\log \left(r_{t} / r_{\mathrm{c}}\right)$; a "c" denotes a core-collapsed cluster.

${ }^{2}$ Refers to the metallicity of the dominant population.

${ }^{3}$ We present evidence of the presence of blue hook stars.

${ }^{4}$ A cluster suffering severe differential reddening.

The photometric calibrations were defined using standard UBVI stars from Landolt (1992). Secondary standard stars from Stetson (http://cadcwww.hia.nrc.ca/standards/) provided a larger number of standards. In a few cases these belonged to the same globular clusters to be calibrated. However, Stetson's fields lack $U$ measurements, hence, in calibrating $U$ data only Landolt $U$ standards were used. Calibration uncertainties in the $U B V I$ filters are estimated to be $0.06,0.03$, 0.03 and 0.04 respectively. In deriving the calibration equations we assumed the following extinction coefficients for La Silla: $K_{U}=0.50, K_{B}=0.23, K_{V}=0.16$ and $K_{I}=0.07$. The small number of $U$ standards makes the calibration of the $U$ data rather uncertain. In particular, the possible presence of nonlinear relations between instrumental and standard magnitudes for very hot (blue stars) could not be checked.

The NTT run (May 31, 2003) was specifically designed to obtain high quality UV diagrams to check the existence of the second- $U$ jump in a number of clusters. At the NTT we used the SUperb-Seeing Imager (SUSI2). The measured pixel scale of SUSI2 is 0 .' 085 arcsec. Each chip of the $2 \times 1$ mosaic covers a field of $5.5 \times 2.7 \mathrm{arcmin}^{2}$. The seeing conditions were relatively good (below 1 .' 3 ), but unfortunately, this night was non-photometric. From this run, we present excellent, but uncalibrated CMDs for three clusters (NGC 5986, NGC 6566 and NGC 6175), i.e. the NTT data will be used only for comparative purposes, i.e. no quantitative descriptions will be drawn from these diagrams.

Lastly, the ground-based color-magnitude diagrams presented in this paper (e.g. Fig. 1) do not necessarily show all the collected photometric data. Indeed, to avoid high crowding conditions near cluster center and outskirt field contamination, each stellar catalog has been obtained by imposing a selection on: (i) the mean value of the image-shape statistics, (SHARP, normally between +0.5 and -0.5 ); (ii) photometric errors; and (iii) radius from the cluster center.

\subsection{HST data}

We searched the HST archive for $F 336 W$ and optical observations of all EHB globular clusters. The importance of multiple dithering (which allows a better derivation of the PSFs, Anderson \& King 2000) and inadequate exposure times reduced the number of useful clusters to only three, namely NGC 2808, NGC 6093 and NGC 6205.

Following the methods by Anderson \& King (2000, 2003), the photometric reduction of the $F 336 W$ and $F 450 W$ observations was carried out with algorithms based on the effective point-spread-function (ePSF) fitting procedure. The essence of the method is a finely sampled PSF of high accuracy, created from images having different offsets. The fit of the ePSF to individual star images gives a precision in position of the order of $\sim 0.02$ pixel, with no systematic errors depending on the location of the star with respect to the pixels boundaries. In principle a good positioning implies good photometry (not visa-versa), and this can be used as a criterion to identify stars with good photometry (as shown in Bedin et al. 2001, 2003). The 16 well-dithered $F 450 \mathrm{~W}$ images of NGC 2808 gave us an ideal case to define the "good-positioning" parameter and served best our purposes of obtaining high accuracy PSFs. This allowed us to extract a "cleaned" $F 336 W, F 450 W$ and F555W catalog.

The instrumental (ePSF) magnitudes were converted to fixed aperture photometry, and then calibrated to the WFPC2 synthetic system using Cols. 2 and 3 from Table 9 in Holtzman et al. (1995). 

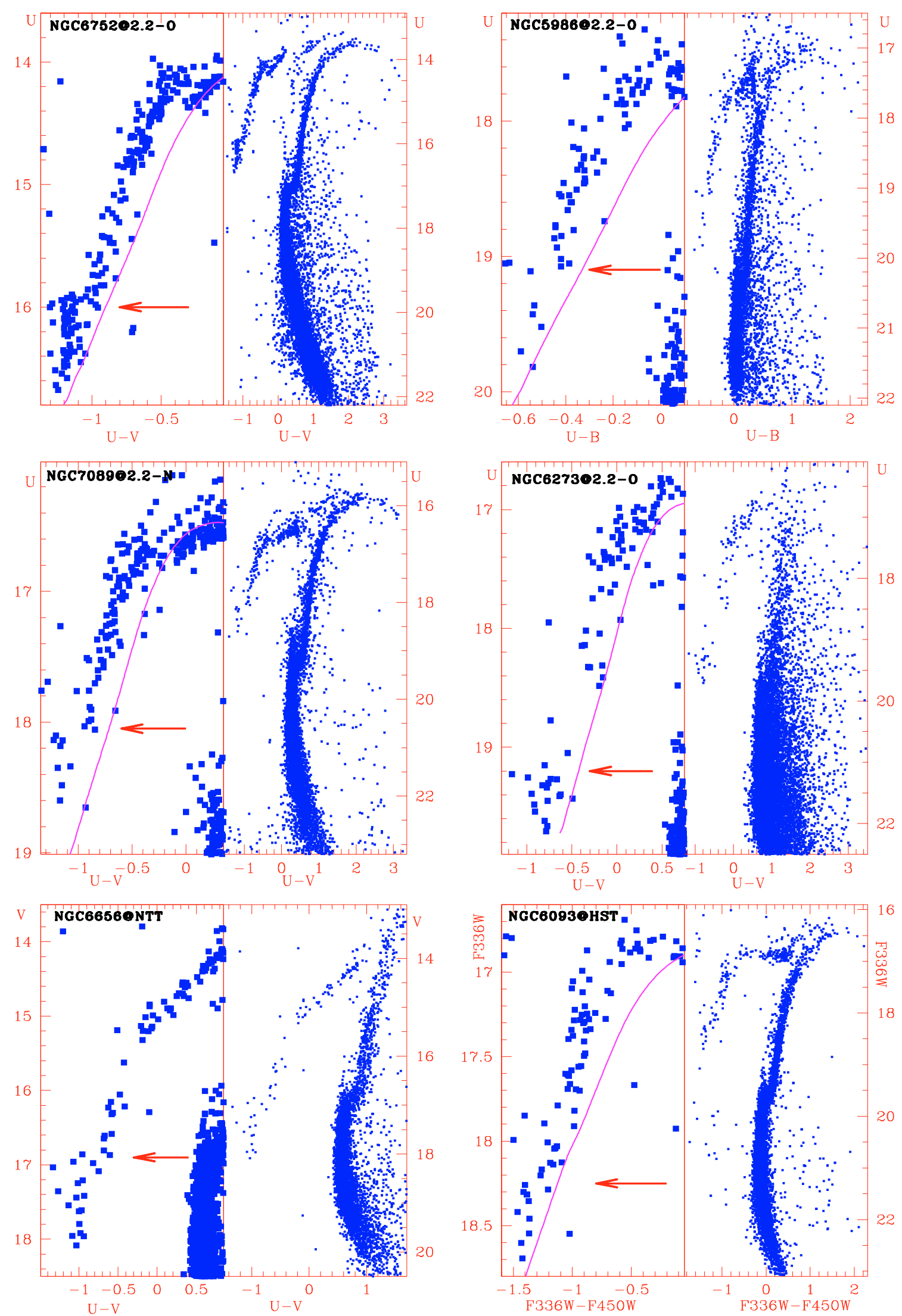

Fig. 1. Near ultraviolet diagrams of 6 EHB clusters. The right panels show the whole CMD (highlighting the high completeness level around the HB level), while left panels show a zoom on the HB stellar distribution, along with a $Z=0.006$ ZAHB model. Arrows roughly mark the onset of the second $U$-jump. 


\section{The near-UV color-magnitude diagrams}

In Fig. 1 we present near-UV CMDs of 6 EHB clusters, namely NGC 6752, NGC 5986, NGC 6273, NGC 7089, NGC 6656, and NGC 6093. In each panel we report the origin of the photometric data. The right panels display the global diagrams, while left panels show a zoom on the EHB to highlight the presence of the second $U$-jump. For data obtained at the $2.2 \mathrm{~m}$ telescope we pay attention to properly identify the adopted filter set (we use the identifier "O" for the old $U$ filter-set and " $\mathrm{N}$ " for the new one): Momany et al. (2003a) have shown how the different transmission curves of the two $U$ filters at the $2.2 \mathrm{~m}$ telescope translate into significant differences in the slope, extension and morphology of blue HB stars. Analyzing the CMDs of Fig. 1 we note the following:

- the global near-UV diagrams show clearly that we are not affected by incompleteness at the faintest level of the HB. The diagrams extend down to $\sim 4.5$ mag below the mainsequence turn offs: i.e. we reach the end of the EHB in all clusters;

- all the CMDs are well populated in stars. Wide-field imaging helped sampling more EHB stars. This is important as we intend to study the extension and the morphology of the hottest, usually less populated part of the HB;

- as indicated by the arrows, there is a discontinuity in the EHB morphology (offset towards bluer colors). The HB is displaced by up to $\sim 0.3 \mathrm{mag}$ in color (depending on the employed color and filters). Apparently, the bluer displaced part extends by $\sim 0.5-0.7$ mag in the $U$-magnitude (again, depending on the employed filters);

- in some clusters, the discontinuity is less evident due to the small number of stars, possible presence of differential reddening and contamination of post-HB stars. Differences in the employed photometric systems also contribute by altering the same features along the HB (Momany et al. 2003a);

- the peculiar cluster NGC 6273, known for its high and differential reddening, also shows the second $U$-jump feature. NGC 6273 is also the cluster with the largest known gap along the HB (see the optical CMD in the HST snapshot photometry by Piotto et al. 1999). This opens the discussion of whether there is a connection between the reported discontinuities and the occurrence of gaps.

We argue that all clusters presented in Fig. 1 show evidence of a discontinuity (second $U$-jump) in their EHB, as that identified in NGC 6752.

\section{The ubiquitous nature of the second $U$-jump?}

In this section, we will verify whether the second $U$-jump is located at the same physical position in all the HBs. If the onset of the second $U$-jump is observed at the same location (e.g. same temperature) in more than one cluster, then one can exclude statistical fluctuations as the cause of its appearance. However, before stating at what temperature this occurs, we mention the difficulty in deriving "photometric temperatures" along the EHB. In Momany et al. (2002) we estimated the onset of the second $U$-jump in NGC $6752(U=15.90$ and $U-V=1.18)$ at $T_{\text {eff }} \sim 23000 \mathrm{~K}$. This estimate was obtained by simply taking the perpendicular projection of where the onset of the second $U$-jump is seen on an adequately shifted ZAHB model. Considering the model uncertainties and the larger bolometric corrections at the very hot end of the EHB, this photometric temperature seemed close to spectroscopic determinations by Moehler et al. (1997). The uncertainty associated with this temperature measurement is of a few thousand degrees.

We derived photometric temperatures for all of the other clusters as for NGC 6752. To avoid other sources of uncertainties due to the distance modulus and reddening, for each cluster we made sure that the G99 jump (clearly visible in all CMDs) was located at the expected temperature of $T_{\text {eff }} \sim 11500 \mathrm{~K}$. The resulting temperature estimates for the second jump are all in the range of $T_{\text {eff }}=21000 \pm 3000 \mathrm{~K}$. Taking into account the uncertainties associated with this measurement, this is an indication that the onset of the second jump is located at the same temperature in all clusters of our sample.

Another indication that the second jump is located at the same temperature in all EHB clusters (presented in Fig. 1) comes from the superposition of the clusters HBs. With the exception of NGC 2808, all our clusters share the same metallicity within 0.2 dex, meaning that the metallicity effects on the HB morphology are expected to be negligible.

The HB superposition is further facilitated by the occurrence of a visible feature at a fixed temperature, irrespective of the examined cluster: the G99 jump at $\sim 11500 \mathrm{~K}$. Although only qualitative, this method showed that the discontinuity in the CMDs occurs at the same location, again suggesting the "ubiquitous" nature of the second jump in all EHBs reaching $T_{\text {eff }} \geq 22000 \mathrm{~K}$. It is important to note that we could not overplot more than three clusters at a time. This is due to the fact that our cluster sample is observed at three different telescopes (NTT, HST and $2.2 \mathrm{~m}$ ) with 4 different filter sets. Examples of such cluster superpositions are shown in Figs. 2, 4 and 5.

\section{The second $U$-jump and the blue hook scenario}

In discussing the occurrence of the second $U$-jump in NGC 6752, Momany et al. (2002) suggested that this feature could be due to a combination of post-ZAHB evolution, diffusion and radiative levitation effects. Having shown the ubiquitous nature of the second $U$-jump in all clusters with EHB exceeding $T_{\text {eff }} \sim 22000 \mathrm{~K}$, in this section we present evidence of a possible connection between the second $U$-jump population and the flash-induced mixing scenario outlined by Brown et al. (2001).

In the past few years, a number of observations (D'Cruz et al. 2000; Whitney et al. 1998; Brown et al. 2001) have clearly shown that the hottest end of the HB in $\omega$ Cen and NGC 2808 is populated by a peculiar class of objects: after Whitney et al. (1998) these were called blue hook stars. A plain theoretical explanation for the evolutionary origin of these stars - the so called He flash induced-mixing scenario - has been provided by Brown et al. (2001). Up to date, this is the most accredited theory on the nature of extremely hot HB stars, and it has recently been supported by both theoretical (Cassisi et al. 2003) and observational efforts (Moehler et al. 2002). 
This scenario (see also Castellani \& Castellani 1993) envisages that, as a consequence of high mass-loss efficiency during the red giant branch evolution (due to enhanced stellar wind and/or dynamical interactions with other stars in the dense cluster core), a star can lose so much envelope mass that it fails to go through a helium flash at the tip of the giant branch, thus evolving toward the white dwarf cooling sequence with an electron-degenerate helium core. Depending on the amount of the residual H-rich envelope mass, the star will initiate Heburning either between the giant branch tip and the bright end of the white dwarf cooling sequence ("early" hot flasher), or along the white dwarf cooling sequence ("late" hot flasher). After the He-flash, these stars will settle on the Zero Age HB (ZAHB). Given their strongly reduced envelope mass, they are much hotter than their counterparts on the canonical ZAHB. In addition, in a late helium flasher structure the convection zone produced by the late $\mathrm{HEF}$ is able to penetrate into the H-rich envelope, thereby mixing $\mathrm{H}$ into the hot He-burning interior (Heflash mixing) where it is burned rapidly. A consequent dredgeup of processed material via both $\mathrm{H}$ - and He-burning enriches the outer envelope with $\mathrm{He}$ and some carbon and nitrogen. According to Brown et al. (2001), these abundance anomalies cause a discontinuous increase of the HB effective temperature at the transition between unmixed and mixed models, producing a gap at the hot end of the HB stellar distribution as indeed observed in the CMD of NGC 2808 of Bedin et al. (2000). At the same time, the changes of the surface chemical composition induced by the He-flash mixing modify the emergent spectral energy distribution, and may explain the fact that these stars appear as sub-luminous objects in far-UV CMD.

\subsection{The case of $\omega$ Centauri}

In Fig. 2 we present a high quality wide-field near-UV CMD of $\omega$ Cen. Panel (A) shows a blue HB that extends over $~ 5$ mag in $V$. In particular, the HB extends $\sim 1.6$ mag below the turn off region; a similar behavior is seen in NGC 2808 and NGC 6715 (Figs. 4 and 5). The region between $-0.8 \leq(U-V) \leq-0.5$ is sparsely populated. Two horizontal (dotted) lines mark the only "visible" gap seen along the HB, roughly corresponding to $T_{\text {eff }} \sim 19000-20000 \mathrm{~K}$. This is the same gap seen in the $V$, $(V-I)$ CMD of Moehler et al. (2002). The end of this gap is close to the onset of the "second jump" population. However, this does not imply a direct gap/jump relation. Indeed, the onset of the second jump in NGC 6752 and NGC 7089 is not preceded by any visible gap. In panel (B) we plot the $(U-V)$ color distribution of the HB. The continuous curve is the smoothed, multi-bin, color-distribution as done in Momany et al. (2003b). The depression in between the two peaks (centered at around $(U-V)=0.5$ and 0.7$)$ traces the gap at $10000 \mathrm{~K}$ probably hampered by photometric errors, while the peak centered at $(U-V)=-1.2$ corresponds to the blue hook population, as suggested below.

A zoom on the HB is shown in panels (C) and (D). The HB in panel (C) seems to be divided into 5 different segments: (1) the blue $H B$ segment, which is the part between the hot boundary of the RR Lyrae instability strip $(U-V \simeq 0.4)$ and the G99 jump ( $U-V \simeq 0.0$ ). This segment of the HB is well-reproduced by a canonical $Z=0.0006 \mathrm{ZAHB}$ model; (2) the segment between $-0.8 \leq U-V \leq 0.0$. Stars in this segment reach a maximum displacement from the ZAHB model at $U-V \simeq-0.5$, then steadily re-approach the model, reaching it around $T_{\text {eff }} \sim 20000 \mathrm{~K}$. The same behavior by other clusters is seen in Fig. 1; (3) a group of stars centered on $U-V \simeq-1.0$ and $17.8 \leq V \leq 18.4$, this is probably the second $U$-jump population; (4) a group of stars centered on $U-V \simeq-1.2$ and $18.5 \leq V \leq 19.0$. This is the blue hook population previously identified by Whitney et al. (1998) and D'cruz et al. (2000) in far-UV studies. Blue hook stars are characterized by their high temperatures $\left(T_{\text {eff }} \geq 35000 \mathrm{~K}\right)$, thus they appear hotter/bluer than the ZAHB model stopping at ( $\left.T_{\text {eff }} \geq 31500 \mathrm{~K}\right)$; and lastly (5) a group of AGB-manqué stars obliquely stretched from $U-V \simeq-1.25$ and $V \simeq 18.00$ to $U-V \simeq-1.35$ and $V \simeq 16.70$.

Moehler et al. (2002) listed a number of $\omega$ Cen stars that have the temperature and chemical signature expected for blue hook stars. To better understand the nature of the blue hook stars, we have identified in our photometry of $\omega \mathrm{Cen}$ the Moehler et al. (2002) stars. Eleven out of 12 stars were found. Table 2 lists their corresponding magnitudes in our cata$\log$ along with the effective temperatures, gravities and helium abundances from Moehler et al. (2002). These objects are labeled in the left panel of Fig. 3, while the right panel shows their helium abundance plotted against the $(U-V)$ color in our photometry. Almost all the blue hook stars of the Moehler et al. (2002) sample lie in the bluest and faintest region of the $V$, $(U-V)$ CMD. There are however two exceptions: BC6022 and BC21840 are located near the hot end of the canonical extreme HB, showing rather "cool" $(U-V)$ indices with respect to their reported temperatures. On the other hand, BC6022 seems also to have a low helium content $\left(\log \left(n_{\mathrm{He}} / n_{\mathrm{H}}\right)=\right.$ $-1.78 \pm 0.16)$, probably indicating a lower flash mixing efficiency. Finally, BC8117 has the bluest $(U-V)$ color in the sample, but a low temperature $\left(T_{\text {eff }}=29800 \pm 1000 \mathrm{~K}\right)$ and low He abundance $\left(\log \left(n_{\mathrm{He}} / n_{\mathrm{H}}\right)=-2.30 \pm 0.23\right)$. As already pointed out by Moehler et al. (2002), BC8117 could be the descendant of an early hot flasher. Clearly, with the exception of BC8117, the helium content increases as the $(U-V)$ index decreases. Therefore, the identification of the group of stars centered at $(U-V) \simeq-1.2$ as the blue hook population in $\omega \mathrm{Cen}$ seems appropriate. This also implies that the number of blue hook stars in $\omega$ Cen is far larger ${ }^{1}$. Having clearly distinguished the blue hook population in $\omega$ Cen, in panel (D) we overplot the CMD of NGC 7089 (open squares) on that of $\omega$ Cen. Again, in the matching process we made sure that the G99 jump of the two clusters coincided ${ }^{2}$. NGC 7089 does not possess a blue hook population, and panel (D) shows that its hottest stars,

\footnotetext{
There are 55 stars centered at $U-V \simeq-1.2$ with $18.5 \leq V \leq$ 19.0. This however is a lower limit for blue hook candidates in $\omega$ Cen simply because the diagram we present in Fig. 2 excludes the central, most crowded, 5.5 arcmin from our $34 \times 33$ arcmin catalog of $\omega$ Cen.

2 The two clusters were observed with the same filter-set at the ESO/MPI 2.2 m telescope.
} 


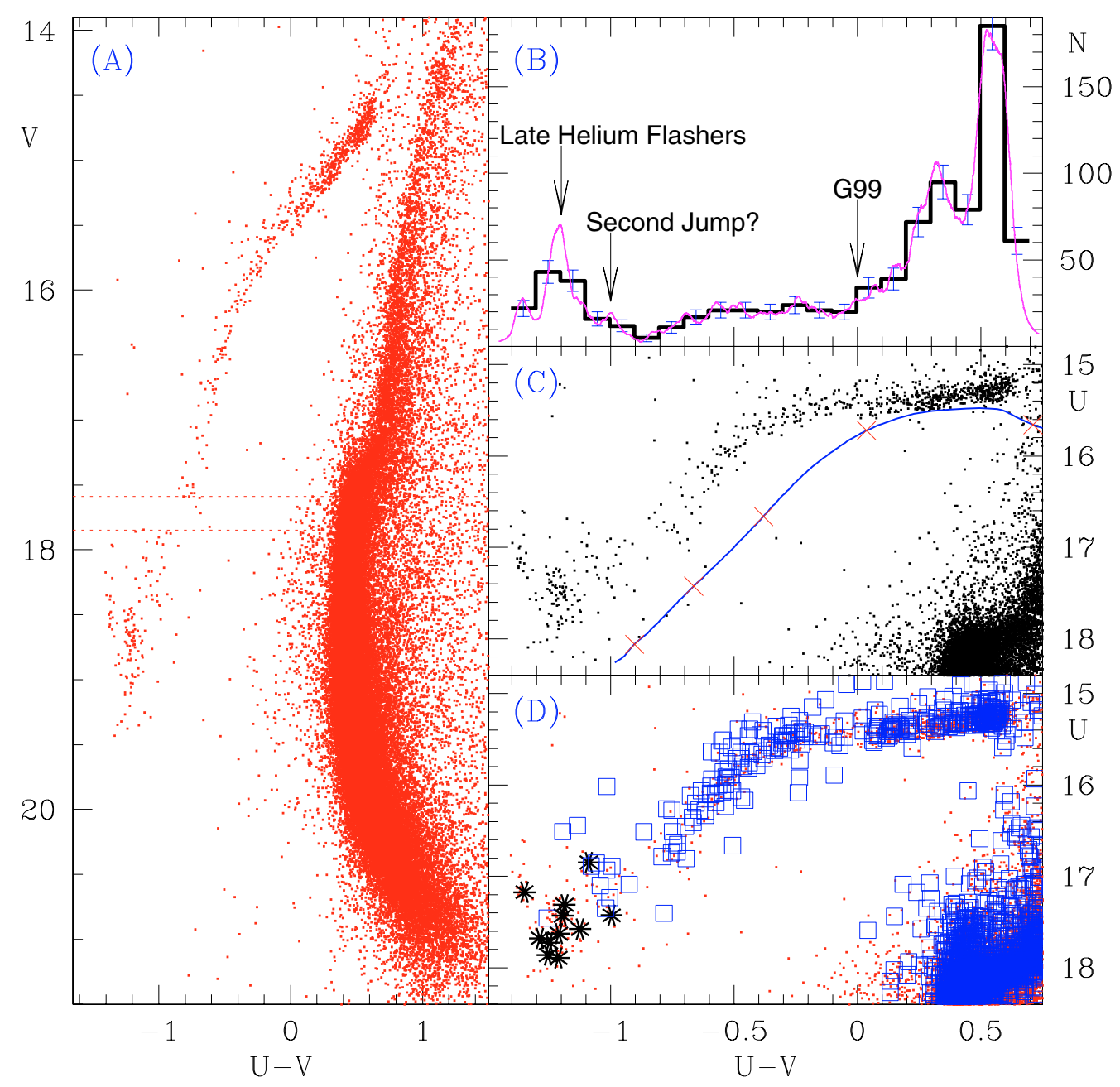

Fig. 2. Near-UV diagrams of $\omega$ Cen: Panel A) presents the global $V,(U-V)$ diagram. The dashed lines mark a possible gap (see also the $V$, $(V-I)$ diagram in Moehler et al. 2002); panel B) shows the $(U-V)$ color distribution histogram, and a corresponding multi-bin, smoothed curve; panel C) shows a zoom on the EHB along with a $Z=0.0006$ ZAHB model. Temperatures of 6000, $11000,16000,23000$ and $30000 \mathrm{~K}$ are marked as crosses; and panel D) shows an overplot of the $U,(U-V)$ diagram of NGC 7089 (open squares) on that of $\omega$ Cen, starred symbols are blue hook spectroscopic stars from the sample of Moehler et al. (2002).

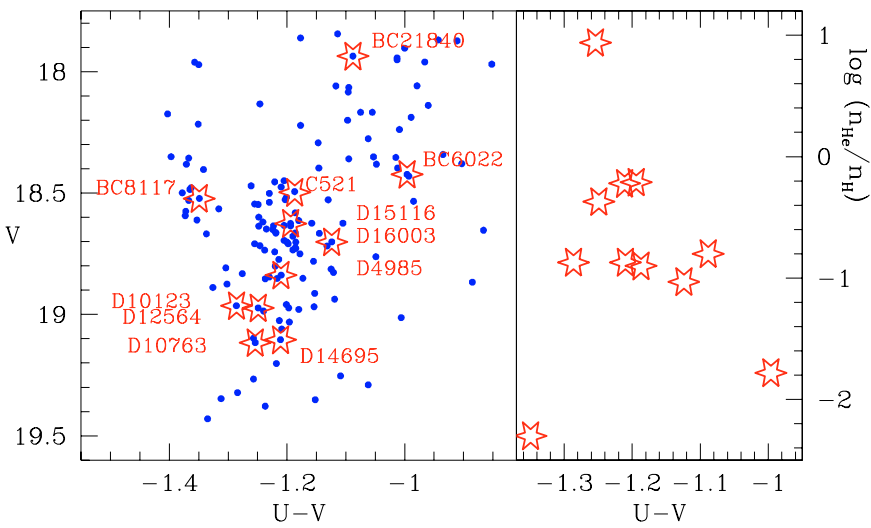

Fig. 3. Left panel shows the $V,(U-V)$ diagrams of $\omega$ Cen, along with the blue hook stars (opens stars) from Moehler et al. (2002). Right panel plots the He abundance (from Moehler et al. 2002) vs. their $(U-V)$ index.

the second $U$-jump population, coincide perfectly with the separated group of stars in $\omega$ Cen that we tentatively identify as the second jump population.
To better understand the relevance of the second jump population in the general picture of extreme $\mathrm{HB}$, and in particular their connection with the He-flash mixing scenario, one must bear in mind the following facts:

- the second $U$-jump population in NGC 6752 extends only to the hot end predicted by canonical HB models. The same applies for the other clusters presented in Fig. 1. None of the clusters presented in Fig. 1 exceeds $T_{\text {eff }} \simeq$ $31500 \mathrm{~K}$. This temperature is lower than the one predicted for blue hook stars in the He-flash mixing scenario ( $T_{\text {eff }} \geq 36000 \mathrm{~K}$ ), hence the two features (blue hook and second jump populations) are quite distinct (see also below);

- second jump stars in NGC 6752 are helium deficient (Moehler et al. 2002);

- the study of Moehler et al. (2002) showed that blue hook stars are indeed hotter $\left(T_{\text {eff }} \geq 35000 \mathrm{~K}\right)$ and are more helium-rich than classical canonical EHB stars. A similar analysis on the blue hook population of NGC 2808 even 
Table 3. Identification of the Moehler et al. (2002) spectroscopically confirmed blue hook stars in our UBVI photometry of $\omega$ Cen. Starred columns are from Moehler et al. (2002).

\begin{tabular}{llllllllll}
\hline \hline $\mathrm{ID}^{*}$ & $\mathrm{RA}_{(2000)}$ & $\operatorname{Dec}_{(2000)}$ & $U$ & $B$ & $V$ & $I$ & $T_{\mathrm{eff}}{ }^{*}$ & $\log g^{*}$ & $\log \left(n_{\mathrm{He}} / n_{\mathrm{H}}\right)^{*}$ \\
\hline BC6022 & $13: 26: 24.936$ & $-47: 33: 23.42$ & 17.427 & 18.365 & 18.423 & 18.620 & $45600 \pm 1300$ & $6.10 \pm 0.14$ & $-1.78 \pm 0.16$ \\
BC8117 & $13: 26: 33.215$ & $-47: 35: 12.56$ & 17.173 & 18.322 & 18.522 & 18.776 & $29800 \pm 1000$ & $5.48 \pm 0.14$ & $-2.30 \pm 0.23$ \\
BC21840 & $13: 27: 25.945$ & $-47: 32: 19.79$ & 16.848 & 17.788 & 17.936 & 18.205 & $35700 \pm 700$ & $5.55 \pm 0.14$ & $-0.80 \pm 0.14$ \\
C521 & $13: 26: 08.834$ & $-47: 37: 12.56$ & 17.310 & 18.314 & 18.497 & 18.690 & $34700 \pm 500$ & $5.90 \pm 0.12$ & $-0.90 \pm 0.09$ \\
D4985 & $13: 25: 14.816$ & $-47: 32: 35.67$ & 17.628 & 18.674 & 18.838 & - & $38400 \pm 800$ & $6.08 \pm 0.16$ & $-0.87 \pm 0.16$ \\
D10123 & $13: 25: 34.268$ & $-47: 29: 50.11$ & 17.678 & 18.807 & 18.964 & 19.225 & $35000 \pm 500$ & $5.82 \pm 0.12$ & $-0.87 \pm 0.09$ \\
D10763 & $13: 25: 35.560$ & $-47: 27: 45.31$ & 17.862 & 18.994 & 19.116 & 19.265 & $35200 \pm 1500$ & $4.35 \pm 0.19$ & $+0.94 \pm 0.14$ \\
D12564 & $13: 25: 41.316$ & $-47: 29: 06.30$ & 17.724 & 18.820 & 18.973 & 19.179 & $36900 \pm 1000$ & $5.60 \pm 0.14$ & $-0.37 \pm 0.09$ \\
D14695 & $13: 25: 46.449$ & $-47: 26: 52.07$ & 17.893 & 18.975 & 19.104 & 19.273 & $41300 \pm 700$ & $6.11 \pm 0.21$ & $-0.22 \pm 0.12$ \\
D15116 & $13: 25: 50.140$ & $-47: 32: 06.29$ & 17.431 & 18.523 & 18.625 & 18.819 & $41500 \pm 1100$ & $6.11 \pm 0.14$ & $-0.21 \pm 0.11$ \\
D16003 & $13: 25: 53.953$ & $-47: 35: 21.64$ & 17.577 & 18.604 & 18.701 & 18.878 & $36300 \pm 600$ & $5.91 \pm 0.12$ & $-1.03 \pm 0.10$ \\
\hline
\end{tabular}

showed carbon enhancement in the most helium-rich stars (Moehler et al. 2004);

- the flash-mixing scenario makes a clear distinction between early and late hot flashers. Unlike the late flashers, early flashers manage somehow to ignite helium in between the giant branch tip and the top of the white dwarf cooling sequence. These also manage to retain a strong enough $\mathrm{H}$-burning shell to prevent any mixing between the helium core and hydrogen envelope. Consequently, no appreciable changes occur in their envelope mass and they do not show altered chemical composition;

- the flash-mixing scenario also predicts that early and late hot flashers should appear quite separated in the CMD and, most interestingly, having a sharp transition between the two populations. The later feature, a sharp transition between early and late flashers, is exactly what is seen in our diagrams. Since there is a sizable population of late helium flashers in $\omega$ Cen, it is reasonable (see also the discussion in Brown et al. 2001) to expect that a significant population of early helium flashers is also present. On theoretical grounds, early helium flashers are predicted to pile up at the end of the extreme HB, nearly indistinguishable from canonical extreme HB stars. Figure 2 shows that the last canonical $\mathrm{HB}$ stars and prior to the onset of late helium flashers (what we call the second $U$-jump population) $d o$ pile up in a narrow color range, exactly as late helium flashers do. We therefore argue that a significant fraction of the second $U$-jump stars in $\omega$ Cen are bona-fide early helium flasher objects.

In summary, on the basis of the evidence above, there is the possibility that the second jump stellar population in $\omega$ Cen contains a significant number of early helium flasher stars. Whereas it is possible to investigate the nature of late helium flasher objects by spectroscopic measurements (see Moehler et al. 2002), there is no way to directly discriminate between early helium flashers and canonical EHB stars, since no differences are expected in their envelope chemical composition. However, it is plausible that the physical mechanism able to enhance the mass loss producing both early and late helium flashers should be the same. One possibility is that mass loss in a giant evolving along the red giant branch is enhanced by the presence of a companion, i.e. in a binary system. It would be worthwhile to observationally investigate the occurrence of binarity among both blue hook stars and second jump stars in $\omega$ Cen, and eventually study the properties of these binaries.

\subsection{The case of NGC 2808}

We have investigated whether $\omega$ Cen is the only cluster possessing both early and late helium flasher stars. The first candidate to verify this hypothesis is NGC 2808, the only other globular cluster with a spectroscopically confirmed blue hook population (see Moehler et al. 2004). Figure 4 presents HST $F 336 W,(F 336 W-F 450 W)$ diagrams of NGC 2808 , done in the same manner as in Fig. 2. With respect to the far-UV diagrams of Brown et al. (2001), panels (A) and (B) show a complete sampling of both hottest and reddest HB stars. Indeed, one can easily detect the (1) RR instability strip centered at $(F 336 W-F 450 W) \simeq 0.2$; (2) G99 jump at $(F 336 W-F 450 W) \simeq-0.35$; (3) gap centered at $F 336 W \simeq$ 17.4 and $(F 336 W-F 450 W) \simeq-1.15$ (Sosin et al. 1997), roughly corresponding to $T_{\text {eff }} \sim 16000 \mathrm{~K}$; (4) second gap located between $-1.65 \leq(F 336 W-F 450 W) \leq-1.30$; (5) late helium flashers (Brown et al. 2001) centered around $(F 336 W-F 450 W) \simeq-1.65$, whose temperature clearly exceeds that of a canonical ZAHB model ${ }^{3}$ as seen in panel $(C)$; and lastly (6) group of post-HB stars extending between $16.20 \leq F 336 W \leq 18.00$ at $(F 336 W-F 450 W) \leq-1.3$.

Comparing Figs. 2 and 4 one notes clear differences in the two color distributions (panel B) and, most interestingly, it is hard to identify the second jump population in NGC 2808 , which should occur at the level of the second gap. This is best seen in panel (D) where we overplot the CMD of NGC 6093 on that of NGC 2808. Again the two CMDs have been matched at the level of the G99 jump. Panel (D) confirms that the second $U$-jump population of NGC 6093 overlaps the gap in the extreme HB of NGC 2808. Although our HB models exclude that

\footnotetext{
3 Evolutionary models adopted in present work have been computed in the framework of canonical stellar evolution, i.e. neglecting any non-canonical physical process.
} 


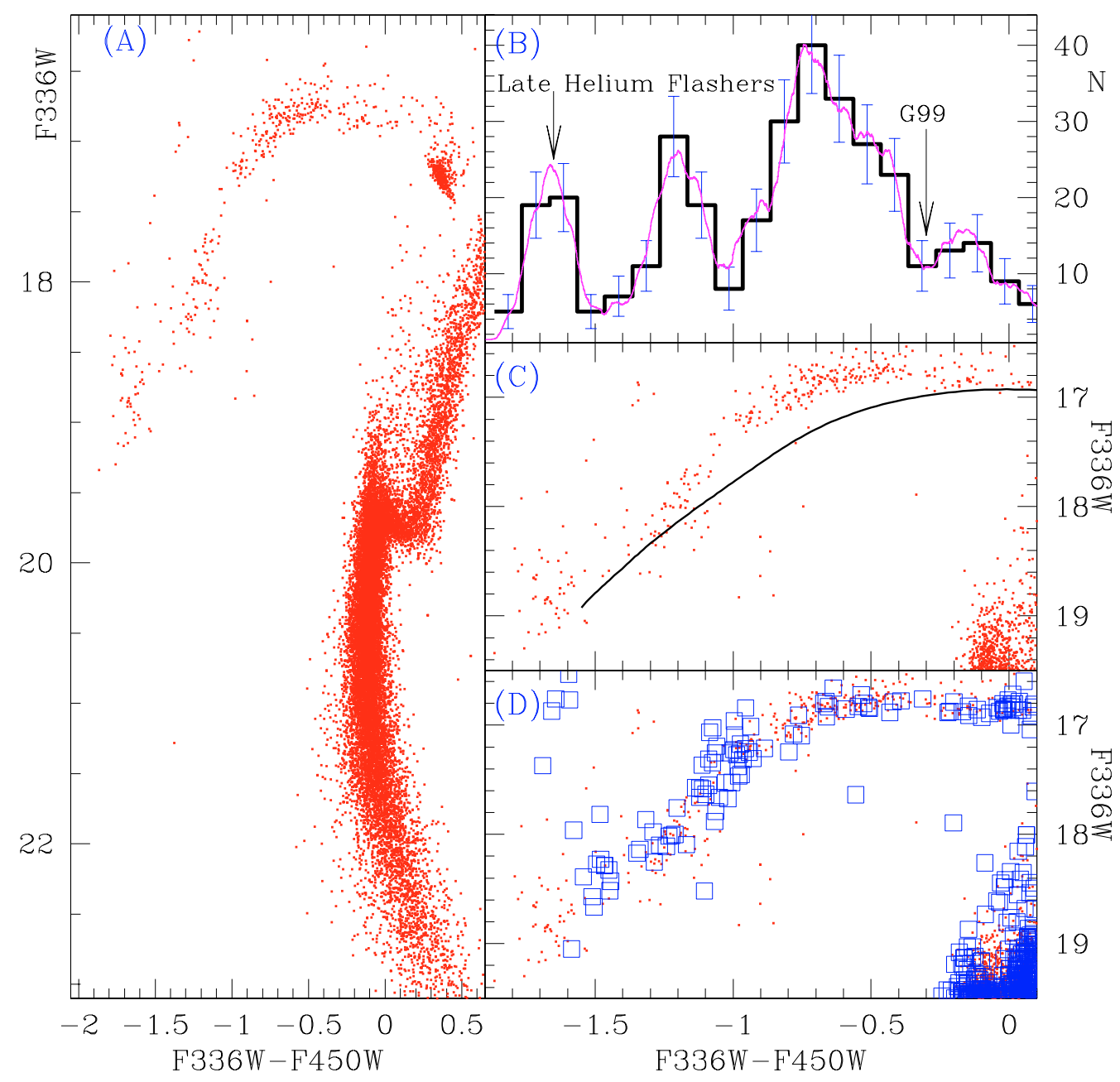

Fig. 4. HST $F 336 W,(F 336 W-F 450 W)$ diagrams of NGC 2808: panel A) presents the global $F 336 W$, $(F 336 W-F 450 W)$ diagram. Panel B) shows the $(F 336 W-F 450 W)$ color distribution histogram, and a corresponding multi-bin, smoothed curve. Panel C) shows a zoom on the EHB along with a $Z=0.001$ ZAHB model; and panel D) shows an overplot of the $F 336 W$, $(F 336 W-F 450 W$ ) diagram of NGC 6093 (open squares) on that of NGC 2808 .

the absence of the second $U$-jump population in NGC 2808 can be due to a metallicity effect which changes the position of the stars along the HB, NGC 2808 is the only cluster with a significantly different metal content with respect to the other clusters in our sample. The immediate conclusion of this comparison is that NGC 2808, besides being the only cluster showing a gap in its extreme $\mathrm{HB}$, it is also the only exception to the general evidence presented in Sect. 4 that all extreme HB clusters reaching $T_{\text {eff }} \geq 22000 \mathrm{~K}$ show the second $U$-jump population.

Assuming the case of $\omega$ Cen (showing both a second jump and late helium flasher populations) as a complete manifestation of the flash-mixed scenario, then one must ask why NGC 2808 seems to skip the formation of the second jump or early helium flasher population. This does not conflict with interpretation given by Brown et al. (2001), who attribute the gap within the extreme HB of NGC 2808 to the dichotomy between the blue hook and canonical extreme HB models. So far, we lack a full explanation of this empirical evidence. However, the absence of the second jump population (or the presence of the second gap) in NGC 2808 is not the only peculiarity of this cluster. Despite the considerable number of both red and blue HB stars (see panel B), and the relatively high mass, NGC 2808 is known to possess only two RR Lyrae stars (Clement \& Hazen 1989). Indeed, the reported "Specific frequency of RR Lyrae variables" in NGC 2808 (0.3) is among the lowest (Harris 1996). The only other cluster showing both red and blue HB stars and such a low RR Lyrae frequency is NGC 6441.

\subsection{The cases of M 54 and NGC 6205}

To further support the suggestion that NGC 2808 is an exception to a scenario in which all extreme HB clusters (reaching $T_{\text {eff }} \geq 23000 \mathrm{~K}$ ) should develop a second jump population, we describe the case of M 54 (NGC 6715). This cluster is in the core of the Sagittarius dwarf galaxy (Ibata et al. 1997). Figure 5 presents our NTT near-UV $\left(\sim 5.5 \times 5.5 \mathrm{arcmin}^{2}\right) \mathrm{di}-$ agram of M 54. As mentioned in Sect. 2.1, the calibration uncertainties in the NTT data (due to a non-photometric night) should not prevent a comparative analysis of the instrumental CMDs. 


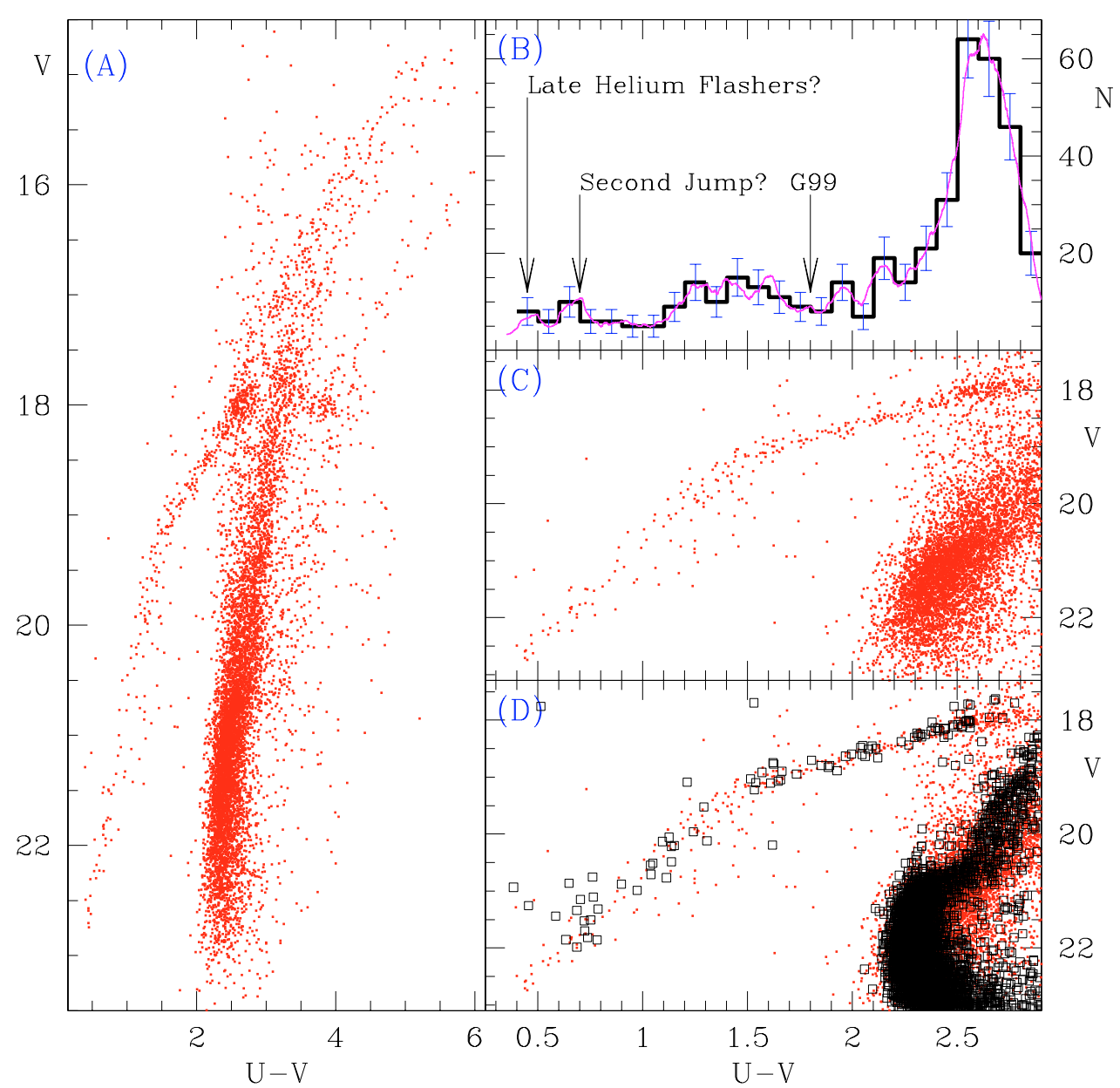

Fig. 5. NTT $V,(U-V)$ diagrams of M54: A) the global diagram; B) the $(U-V)$ color distribution histogram, and a corresponding multi-bin, smoothed curve; C) a zoom on the extreme HB; and D) an overplot of the $V,(U-V)$ diagram of NGC 6656 (open squares) on that of M 54. These are instrumental diagrams.

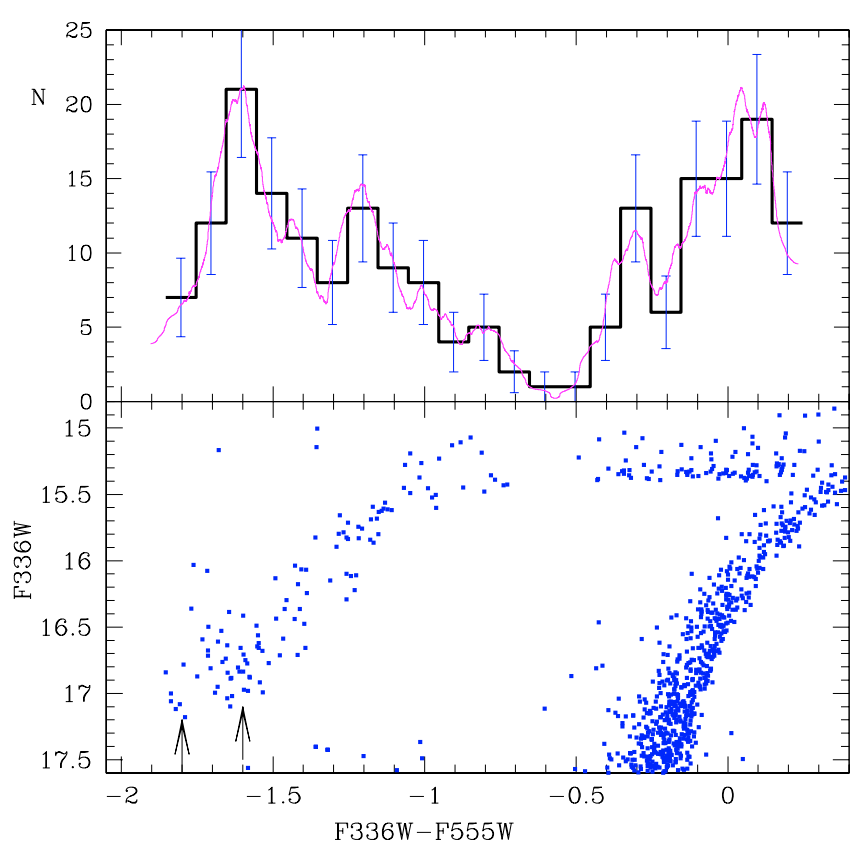

Fig. 6. HST $F 336 W$, $(F 336 W-F 555 W)$ diagram of NGC 6205. Arrows mark the second jump population and a group of hotter stars.
On the one hand, panel (A) shows that the extension of the blue HB below the turn off region is very similar to that of $\omega$ Cen and NGC 2808 ; i.e. $\simeq 1.6 \mathrm{mag}$ in $V$. This suggests the presence of a late helium flasher population in $\mathrm{M} 54^{4}$. The main point we want to emphasize here is shown in panel (D). Overplotting the CMD of NGC 6656 (a cluster possessing a second jump population but no late helium flashers) on M 54, one sees that the location of the second jump population in NGC 6656 overlaps a group of stars in M 54; i.e. M 54 seems to possess both the second jump and late helium flasher populations.

Within the scenario we proposed in Sect. 5, the HB of $\omega$ Cen and M 54 show evidence for both early and late helium flashers, whereas NGC 2808 shows only late flashers. Obviously with such a small sample of stellar systems hosting early and late helium flashers we cannot derive sound conclusions regarding whether the production of both early and late helium flashers is the general rule or if it is an exception.

We also investigated the possible presence of blue hook stars in NGC 6205. Figure 6 presents the HST $F 336 W$, $(F 336 W-F 555 W)$ diagram of NGC 6205 . Arrows mark the onset of the second jump population (see also the diagrams in

\footnotetext{
${ }^{4}$ A similar conclusion has been reached by Rosenberg et al. (2004), based on $B V$ photometry.
} 
Grundahl et al. 1999) and a group of stars, offset towards bluer colors and fainter magnitudes. These could be AGB-manqué stars as well as late helium flashers, and clearly call for a spectroscopic follow-up.

\section{Summary}

We have presented near-UV diagrams for a dozen clusters. Our main result is that the previously reported discontinuity in NGC 6752 (around $T_{\text {eff }} \simeq 23000 \mathrm{~K}$ ) is also present in other extreme HB clusters. The onset of this second $U$-jump in the examined clusters seems also to coincide at a temperature of $\sim 21000 \pm 3000 \mathrm{~K}$. Both these facts strengthen the idea that the second $U$-jump is an indicator of a physical process, acting in all extreme HB clusters, that has yet to be fully understood. We have clearly shown that the second $U$-jump population is photometrically distinct from the hotter blue hook stars, and brought evidence that the two chemically different populations can co-exist in objects like $\omega$ Cen and M 54. In the flash mixing scenario, early helium flashers are expected to pile up at the end of the extreme $\mathrm{HB}$, and this is exactly what the second jump population seems to show. Hence, we suggest that the second jump population contains a significant number of early helium flashers.

\subsection{Final remarks and future work}

One of the main difficulties in studying the HB star distribution is providing a satisfactory, quantitative description of its morphology. To this end, many HB morphology parameters were introduced (Fusi Pecci et al. 1993; Buonanno et al. 1997; Catelan et al. 1998; Piotto et al. 1999 and references therein) to describe the distribution of HB stars in color/temperature, measure their maximum extension, and reveal peaks and gaps. It remains however, that the majority of these studies was based on $B V$ CMDs, clearly not an ideal plane for detailed studies of the HB (Ferraro et al. 1998).

In light of the growing number of UV CMDs, and recent photometric findings (the G99 and second $U$-jumps, and blue hook stars) we revisit the HB morphology in the UV plane. In brief, the analysis of the HBs in 7 clusters lead us to suggest that the HB in UV CMDs can be envisaged as the sum of discrete segments. This is not a new idea (Buonanno et al. 1985). However the new observations in the UV plane and the larger sample of HB stars unveil a number of discrete branches in the HB previously undisclosed. To better explain this fact we rely on Fig. 7, showing a combination of UV CMDs. A more detailed and complete analysis will be the subject of a future work. Figure 7 allows us to suggest the following:

1. the discontinuities (gaps or jumps) at $T_{\text {eff }} \sim 10000 \mathrm{~K}$ and $11500 \mathrm{~K}$ occur in all blue HB clusters, in particular, the G99 jump coincides with the HB endpoint of many blue HB clusters and Local Group dwarf galaxies;

2. there are two other main discontinuities at $T_{\text {eff }} \sim 16000 \mathrm{~K}$ and $21000 \mathrm{~K}$, marking the endpoints of $\mathrm{HB}$ reaching these temperature.
Point (1) confirms the already known ubiquitous nature of the G99 jump, and suggests a similar ubiquity of the gap at $T_{\text {eff }} \sim 10000 \mathrm{~K}$ (the gap at $B-V$ about zero in the Caloi 1999 terminology). Similarly, Ferraro et al. (1998) also suggested that the gaps at $T_{\text {eff }} \sim 10000 \mathrm{~K}$ and $11500 \mathrm{~K}$ (G0 and G1 in their terminology) occur in many but not all clusters. Hence, the novelty in point (1) lies in suggesting the ubiquity of the gap at $T_{\text {eff }} \sim 10000 \mathrm{~K}$, and this mainly relies on the optical CMDs of the HST snapshot (Piotto et al. 2002) and panels (B)-(D) of Fig. 7. The three panels propose three ways in which clusters populate the blue HB (i.e., the HB extending from the bluest boundary of the RR instability strip to the onset of the G99 jump) around the $T_{\text {eff }} \sim 10000 \mathrm{~K}$ gap.

The first case (panel A overplotting NGC 7099 on NGC 6752) is the most frequent, that is, a more or less uniform distribution on the two parts surrounding the gap at $T_{\mathrm{eff}} \sim$ $10000 \mathrm{~K}$. Relying on the optical CMDs of the HST snapshot (Piotto et al. 2002) and our UV diagrams we count 34 clusters with a metallicity range between $[\mathrm{Fe} / \mathrm{H}]=-1.27$ (NGC 5904) and $[\mathrm{Fe} / \mathrm{H}]=-2.29$ (NGC 5053) which uniformly populate only this part of the HB (i.e. having no red HB stars and no HB stars hotter than the G99 jump). Most of these clusters (e.g. NGC 7099) show a clear gap at $T_{\text {eff }} \sim 10000 \mathrm{~K}$. On the other hand, we note that Local Group dwarf spheroidals are also representative of a uniformly populated blue HB. The diagrams of Ursa Minor (Carrera et al. 2002) and Sculptor (Hurley-Keller et al. 1999) are examples of blue HB extending only in this temperature range, possibly showing the gap at $T_{\text {eff }} \sim 10000 \mathrm{~K}$ gap.

The second and third case (panels B and C) is when clusters preferentially populate one side of the $T_{\text {eff }} \sim 10000 \mathrm{~K}$ gap; either the hotter or the cooler side. NGC $7078^{5}$ and NGC 5466 (see CMD in Buonanno et al. 1985) are examples of blue $\mathrm{HB}$ extending from the RR instability strip and stopping at $T_{\text {eff }} \sim 10000 \mathrm{~K}$; i.e. populating only the right side of the gap. On the other hand NGC 288 (panel C) shows the opposite behavior; populating the part between $T_{\text {eff }} \sim 10000 \mathrm{~K}$ and $11500 \mathrm{~K}$; i.e. populating the left side of the $T_{\text {eff }} \sim 10000 \mathrm{~K}$ gap. Overall, the 3 proposed distribution modalities might explain previous difficulties in ascertaining the ubiquity of the gap at $\sim 10000 \mathrm{~K}$ (see discussions in Ferraro et al. (1998) on the varying width of the $T_{\mathrm{eff}} \sim 10000 \mathrm{~K}$ gap from cluster to cluster).

We did not find any cluster with HB extending beyond the G99 jump which does not possess blue HB stars with $T_{\text {eff }} \leq 11500 \mathrm{~K}$. In other words, all the clusters with hot HB stars $\left(T_{\text {eff }} \geq 11500 \mathrm{~K}\right)$ do have stars in the region between the RR Lyrae instability strip and the G99 jump.

We do not have an explanation for such a complex behavior in this part of the HB. However, the presented evidence implies that the discontinuities at $T_{\text {eff }} \sim 10000 \mathrm{~K}$ and $11500 \mathrm{~K}$ are presumably present in all clusters. Whereas the G99 jump can be easily discerned in UV CMDS, the gap at $T_{\text {eff }} \sim 10000 \mathrm{~K}$ might be hampered by photometric errors and post-HB

\footnotetext{
5 Note that the group of stars distributed on the right side of the blue HB clump are probably variable stars (see Zheleznyak \& Kravtosov 2003) caught at random phase.
} 


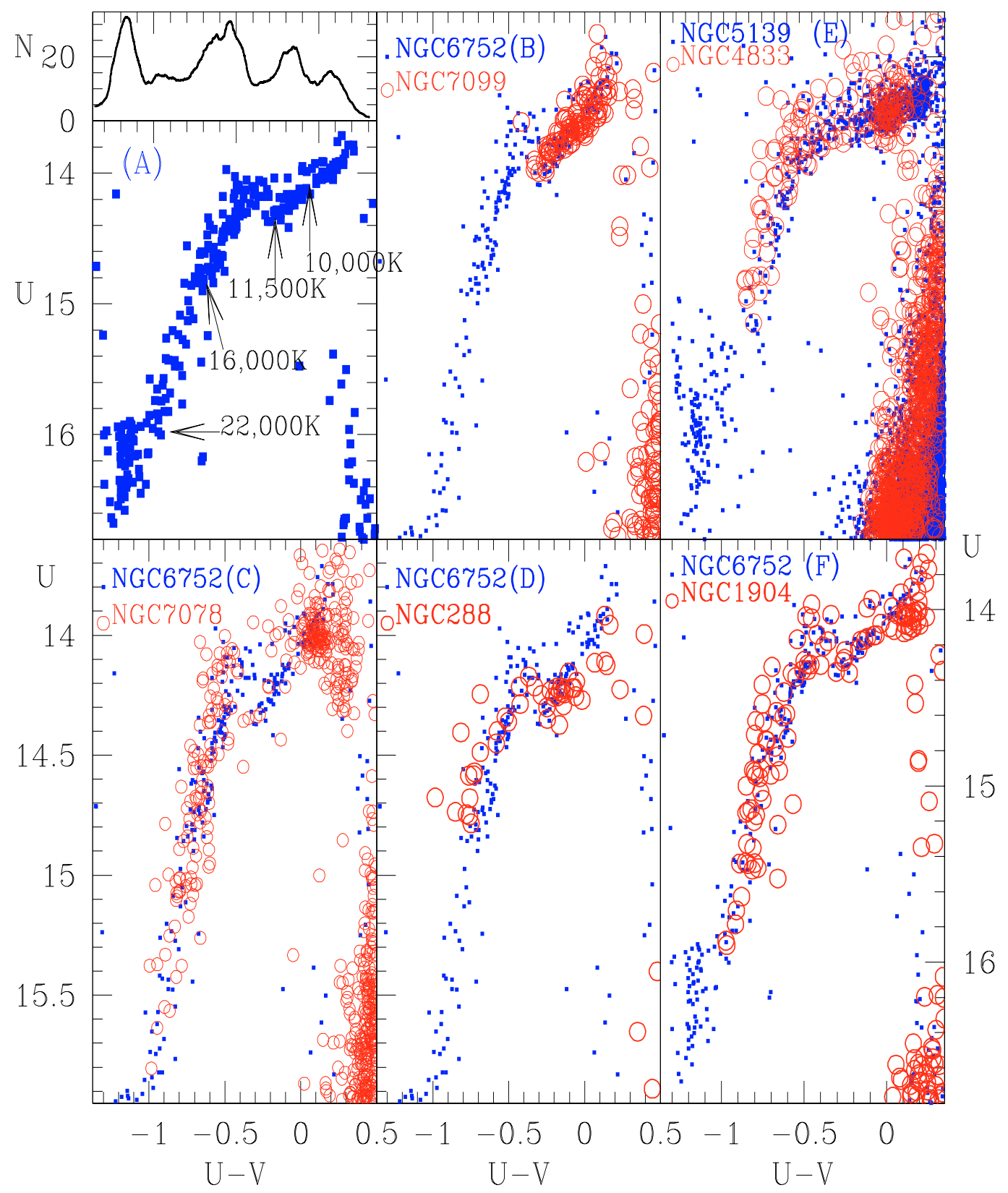

Fig. 7. The segmented nature of the blue HB. Panel A) shows a zoom on the HB of NGC 6752 along with its color distribution and the identification of specific temperatures. Panels B) through F) show CMDs superpositions of NGC 7078, NGC 7099, NGC 288 and NGC 1904 on NGC 6752, except for panel E) where we overplot NGC 4833 on $\omega$ Cen.

evolution. Moreover, given the high frequency of globular clusters and dwarf galaxies populating only this specific range of the blue HB (RR instability strip-onset of the G99 jump), this group of objects might represent the "standard" HB morphology in the metal-poor regime. As discussed in Buonanno et al. (1985), one way around the second parameter debate can be to isolate certain groups of clusters (with a substantial similarity in some of their basic properties) and then explore the effects of any other difference the clusters in the group have. Clusters/dwarf galaxies showing only the blue HB (between the RR instability strip and the onset of the G99 jump) are most probably a separate group, within which one can search for "similarities".
What is so special in the group of clusters (e.g. NGC 7099) ending their blue HB exactly at the onset of the G99 jump at $T_{\text {eff }} \sim 11500 \mathrm{~K}$ ? The occurrence of the G99 jump was explained as the aftermath of radiative levitation that causes a substantial increase in the metal content of the outermost layers. Radiative levitation is possible after the disappearance of the envelope convective layers located across the $\mathrm{H}$ and HeI ionization regions at $T_{\text {eff }} \sim 10000 \mathrm{~K}$ and $11000 \mathrm{~K}$ respectively (Caloi 1999; Sweigart 2000). Hence, both the gap at $T_{\text {eff }} \sim 10000 \mathrm{~K}$ and the G99 jump at $\sim 11500 \mathrm{~K}$ can be attributed to atmospheric effects. If we adopt this explanation for the two discontinuities, and given their omni-presence in different environments, then systems with only blue HBs ending 
just before the onset of the G99 jump can be seen as clusters in which a "standard" mass loss mechanism takes place. The net product of this "standard" mass loss on the red giant branch is always a HB star with an envelope, massive enough, to possess an extended convective region.

Similarly, for clusters with $\mathrm{HB}$ extending beyond $T_{\text {eff }} \sim$ $11500 \mathrm{~K}$, we have identified two truncation points in the blue tail: one at at $T_{\text {eff }} \sim 16000 \mathrm{~K}$ and a second at $\sim 21000 \mathrm{~K}$.

Panel (D) shows that NGC 288 ends at $T_{\text {eff }} \sim 16000 \mathrm{~K}$. As shown in the color distribution of the HB in NGC 6752 (panel A), this temperature corresponds to a marked decrease in the stellar counts in NGC 6752. On the other hand, this is also the temperature at which the first of the NGC 2808 gaps occurs (Bedin et al. 2000). Hence, besides the G99 jump, $T_{\text {eff }} \sim 16000 \mathrm{~K}$ seems to mark another endpoint. Panels (E) and $(\mathrm{F})$ indicate another important endpoint: the onset of the second $U$-jump. Obviously one cannot rely on few stars to mark the end of the HB blue tail of NGC 1904 and NGC 4833, however the coincidence with the onset of the second jump is rather tempting. In this regard, it is of great interest to further investigate a possible relation between the second jump population and the early helium flashers. As a working hypothesis, if all the second jump stars were to be early helium flashers, it would imply that stars hotter than $T_{\text {eff }} \sim 21000 \mathrm{~K}$ have a different physical origin (flash-mixing scenario?) with respect to "cooler" HB stars (produced by standard mass-loss mechanisms). This possibility would have significant implications for our understanding of the complicated second parameter problem.

The overall picture of the HB in UV diagrams seems rather segmented. The endpoints that define these segments may be acting as markers, highlighting the signature of different physical processes working in $\mathrm{HB}$ stars. The origin of the $T_{\text {eff }} \sim$ $10000 \mathrm{~K}$ and $\sim 11500 \mathrm{~K}$ discontinuities seems to be related to the disappearance of the convective envelope layers located across the $\mathrm{H}$ and HeI ionization regions (Caloi 1999). In this paper we have shown that the discontinuity at $T_{\text {eff }} \sim 21000 \mathrm{~K}$ can be related to the presence of early helium flashers. For the endpoint at $T_{\text {eff }} \sim 16000 \mathrm{~K}$ we do not yet have an explanation.

Acknowledgements. We warmly thank R. Gratton for useful discussions, and H. Navasardyan for her help in calibrating WFI data. We acknowledge financial support of the MIUR (PRIN 2001) and ASI.

\section{References}

Anderson, J., \& King, I. R. 2000, PASP, 112, 1360

Anderson, J., \& King, I. R. 2003, PASP, 115, 113

Bedin, L. R., Piotto, G., Zoccali, M., et al. 2000, A\&A, 363, 159

Bedin, L. R., Anderson, J., King, I. R., \& Piotto, G. 2001, ApJ, 560, L75

Bedin, L. R., Piotto, G., King, I. R., \& Anderson, J. 2003, AJ, 126, 247

Behr, B. B., Cohen, J. G., McCarthy, J. K., \& Djorgovski, S. G. 1999, ApJ, 517, L135

Behr, B. B., Djorgovski, S. G., Cohen, J. G., et al. 2000, ApJ, 528, 849

Buonanno, R., Corsi, C. E., \& Fusi Pecci, F. 1985, A\&A, 145, 97
Buonanno, R., Corsi, C., Bellazzini, M., Ferraro, F. R., \& Pecci, F. F. 1997, AJ, 113, 706

Brown, T. M., Sweigart, A. V., Lanz, T., Landsman, W. B., \& Hubeny, I. 2001, ApJ, 562, 368

Caloi, V. 1999, A\&A, 343, 904

Carrera, R., Aparicio, A., Martínez-Delgado, D., \& Alonso-García, J. 2002, AJ, 123, 3199

Cassisi, S., Schlattl, H., Salaris, M., \& Weiss, A. 2003, ApJ, 582, L43

Castellani, M., \& Castellani, V. 1993, ApJ, 407, 649

Catelan, M., Borissova, J., Sweigart, A. V., \& Spassova, N. 1998, ApJ, 494, 265

Clement, C. M., \& Hazen, M. L. 1989, AJ, 97, 414

D’Cruz, N. L., Dorman, B., Rood, R. T., \& O’Connell, R. W. 1996, ApJ, 466, 359

D’Cruz, N. L., O'Connell, R. W., Rood, R. T., et al. 2000, ApJ, 530, 352

Ferraro, F. R., Paltrinieri, B., Pecci, F. F., Rood, R. T., \& Dorman, B. 1998, ApJ, 500, 311

Fusi Pecci, F., Ferraro, F. R., Bellazzini, M., et al. 1993, AJ, 105, 1145

Greggio, L., \& Renzini, A. 1990, ApJ, 364, 35

Grundahl, F., Catelan, M., Landsman, W. B., Stetson, P. B., \& Andersen, M. I. 1999, ApJ, 524, 242 [G99]

Harris, W. E. 1996, AJ, 112, 1487

Holtzman, J. A., Burrows, C. J., Casertano, S., et al. 1995, PASP, 107, 1065

Hurley-Keller, D., Mateo, M., \& Grebel, E. K. 1999, ApJ, 523, L25

Ibata, R. A., Wyse, R. F. G., Gilmore, G., Irwin, M. J., \& Suntzeff, N. B. $1997, A J, 113,634$

Landolt, A. U. 1992, AJ, 104, 372

Moehler, S., Heber, U., \& Rupprecht, G. 1997, A\&A, 319, 109

Moehler, S., Sweigart, A. V., Landsman, W. B., \& Heber, U. 2000, A\&A, 360, 120

Moehler, S., Sweigart, A. V., Landsman, W. B., \& Dreizler, S. 2002, A\&A, 395, 37

Moehler, S., Sweigart, A. V., Landsman, W. B., Hammer, N. J., \& Dreizler, S. 2004, A\&A, 415, 313

Momany, Y., Piotto, G., Recio-Blanco, A., et al. 2002, ApJ, 576, L65

Momany, Y., Cassisi, S., Piotto, G., et al. 2003a, A\&A, 407, 303

Momany, Y., Ortolany, S., Held, E. V., et al. 2003b, A\&A, 402, 607

Piotto, G., \& Zoccali, M. 1999, A\&A, 345, 485

Piotto, G., Zoccali, M., King, I. R., et al. 1999, AJ, 118, 1727

Piotto, G., King, I. R., Djorgovski, S. G., et al. 2002, A\&A, 391, 945

Recio-Blanco, A., Piotto, G., Aparicio, A., \& Renzini, A. 2002, ApJ, 572, L71

Rich, R. M., Sosin, C., Djorgovski, S. G., et al. 1997, ApJ, 484, L25

Rosenberg, A., Saviane, I., Piotto, G., \& Aparicio, A. 1999, AJ, 118, 2306

Rosenberg, A., Recio-Blanco, A., \& Garciá-Marin, M. 2004, ApJ, 603, 135

Sandage, A., \& Wildey, R. 1967, ApJ, 150, 469

Sosin, C., Dorman, B., Djorgovski, S. G., et al. 1997, ApJ, 480, L35

Stetson, P. B. 1994, PASP, 106, 250

Sweigart, A. V. 1997, ApJ, 474, L23

Sweigart, A. 2000, Mixing and Diffusion in Stars: Theoretical Predictions and Observational Constraints, 24th meeting of the IAU, Joint Discussion 5, August, Manchester, England, 5

Valdes, F. G. 1998, ASP Conf. Ser. 145: Astronomical Data Analysis Software and Systems VII, 7, 53

Whitney, J. H., Rood, R. T., O'connell, R. W., et al. 1998, ApJ, 495, 284

Zheleznyak, A. P., \& Kravtsov, V. V. 2003, Astron. Lett., 29, 599 